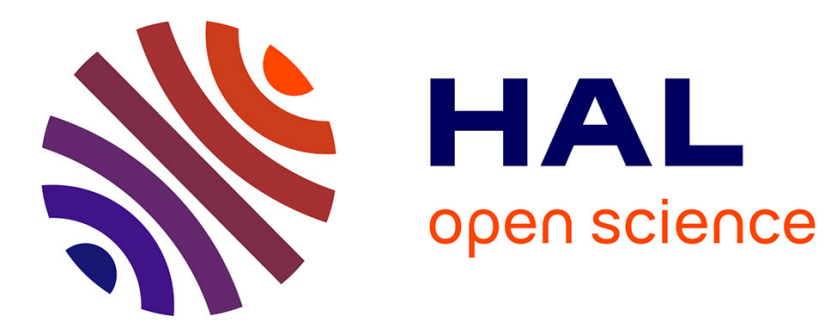

\title{
Analytic wavelets for multivariate time series analysis
}

Irène Gannaz, Sophie Achard, Marianne Clausel, François Roueff

\section{To cite this version:}

Irène Gannaz, Sophie Achard, Marianne Clausel, François Roueff. Analytic wavelets for multivariate time series analysis. SPIE Optical Engineering + Applications, Aug 2017, San Diego, Californie, United States. pp.1-8, 10.1117/12.2272928 . hal-01618447

\section{HAL Id: hal-01618447 https://hal.science/hal-01618447}

Submitted on 20 Mar 2019

HAL is a multi-disciplinary open access archive for the deposit and dissemination of scientific research documents, whether they are published or not. The documents may come from teaching and research institutions in France or abroad, or from public or private research centers.
L'archive ouverte pluridisciplinaire HAL, est destinée au dépôt et à la diffusion de documents scientifiques de niveau recherche, publiés ou non, émanant des établissements d'enseignement et de recherche français ou étrangers, des laboratoires publics ou privés. 


\title{
Analytic wavelets for multivariate time series analysis
}

\author{
Irène Gannaz ${ }^{\mathrm{a}}$, Sophie Achard ${ }^{\mathrm{b}}$, Marianne Clausel $^{\mathrm{c}}$, and François Roueff ${ }^{\mathrm{d}}$ \\ aUniversité de Lyon, CNRS UMR 5208, INSA de Lyon, Institut Camille Jordan, France \\ ${ }^{\mathrm{b}} \mathrm{CNRS}$, Univ. Grenoble Alpes, GIPSA-lab, Grenoble, France \\ 'Univ. Grenoble Alpes, LJK, Grenoble, France \\ dLTCI, Télécom-Paristech, Université Paris-Saclay, France
}

\begin{abstract}
Many applications fields deal with multivariate long-memory time series. A challenge is to estimate the long-memory properties together with the coupling between the time series. Real wavelets procedures present some limitations due to the presence of phase phenomenons. A perspective is to use analytic wavelets to recover jointly long-memory properties, modulus of long-run covariance between time series and phases. Approximate wavelets Hilbert pairs of Selesnick (2002) fullfilled some of the required properties. As an extension of Selesnick (2002)'s work, we present some results about existence and quality of these approximately analytic wavelets.
\end{abstract}

Keywords: Complex wavelets, multivariate time series

\section{INTRODUCTION}

An analytic wavelet is a complex wavelet such that its Fourier transform is null for negative frequency. By the Paley-Wiener theorem, it is impossible to find wavelets with both Fourier transform with null negative frequency and compact support. Indeed, compact support is an important property that is usually desirable in practice for wavelets. Moreover, the analyticity property enables to capture information for the phase and not only the module. ${ }^{1}$ Previous approaches have been developped by constructing approximately analytic wavelets. ${ }^{1}$ An example of such wavelets is based on Hilebert-pairs constructed using a common-factor design. ${ }^{2,3}$ Despite their usefulness in practice, few theoretical results have been described. In particular, to our knowledge, their existence is not proved. These wavelets depend on two integer parameters $L$ and $M$ that correspond respectively to the order of the analytic approximation and the number of null moments.

Our contribution in this paper is to demonstrate that the construction of such wavelets is valid by proving their existence for any parameters $L, M \geqslant 1$. In addition, an exact formula is obtained with an explicit expression. This expression allows us to evaluate the analyticity approximation of the wavelets, i.e. to control the presence of the negative frequency with respect to the parameters $L$ and $M$.

After the description of the theoretical aspects of common-factor wavelets, their practical use is illustrated for the analysis of multivarite time series with long memory effect. Such data are found in a large number of applications, for example finance, ${ }^{4,5}$ internet traffic analysis, ${ }^{6}$ physical sciences, ${ }^{7,8}$ geosciences ${ }^{9}$ and neuroimagery. ${ }^{10}$ In multivarite time series, a phase phenomenon occurs, which plays a crucial role in the modeling of interdependence between the components.

In addition, when dealing with long memory processes, frequency analysis using periodogram or scalogram is particularly adequate for second order stationary processes. ${ }^{6,11}$ These analyses are usually using

\footnotetext{
Further author information: (Send correspondence to I.G.)

I.G.: E-mail: irene.gannaz@insa-lyon.fr

S.A.: E-mail: Sophie.Achard@gipsa-lab.grenoble-inp.fr

M.C.: E-mail: marianne.clausel@univ-grenoble-alpes.fr

F.R.: E-mail: francois.roueff@telecom-paristech.fr
} 
low frequencies and the phase is not studied. However, few papers ${ }^{12,13}$ showed that the phase is important even for low frequencies. In this context, an approach based on (approximately) analytic wavelets may be useful. Indeed we illustrate the ability of (approximately) analytic wavelets to capture the phase in a simple example. $^{12}$

The paper is organised in two parts. The first part is dedicated to the construction of approximately analytic wavelets by common-factor design. The existence of the construction is detailled by giving an explicit expression of the common-factor. In addition, this allows us to quantify the analytic approximation. The second part presents the good behaviour of this approach when dealing with multivariate long-memory model $M(\mathbf{d}) .{ }^{12,14}$

\section{CONSTRUCTION AND EXISTENCE OF THE WAVELET BASIS}

The challenging problem in the construction of a wavelet basis is the impossibility to get a Hilbert pair with finite response filters (FIR). A choice is to relax the analyticity and search for an approximation of the Hilbert-pair condition. ${ }^{1}$ Based on the common-factor design, ${ }^{1}$ we detail the construction in this part.

\subsection{Definition}

Define $\psi_{G}$ and $\psi_{H}$ two real wavelets functions. They form a Hilbert pair when their Fourier transforms, denoted respectively $\widehat{\psi}_{G}$ and $\widehat{\psi}_{H}$, satisfy

$$
\widehat{\psi}_{G}(\omega)=-i \operatorname{sign}(\omega) \widehat{\psi}_{H}(\omega) .
$$

Let $g_{0}$ and $h_{0}$ denote the pass-band filters associated to $\psi_{G}$ and $\psi_{H}$. Introduce $G_{0}$ and $H_{0}$ the transfer functions of $g_{0}$ and $h_{0}$ obtained by a $z$-transform; for example, $G_{0}(z)=\sum_{n} g_{0 n} z^{-n}$. The property (1) cannot be satisfied with FIR filters. ${ }^{15}$ The only choice is then to define FIR filters that verifies approximately (1), that is,

$$
G_{0}\left(\mathrm{e}^{\mathrm{i} \omega}\right) \sim H_{0}\left(\mathrm{e}^{\mathrm{i} \omega}\right) \mathrm{e}^{-\mathrm{i} \omega / 2} \text { when } \omega \rightarrow 0 .
$$

Such a construction has already been proposed, ${ }^{15}$ where the practical construction of the wavelets is described but the existence is not acquired. Hence the quality of approximation in (2) is not explicit. Our contribution is to derive these two points in the following of the paper.

\subsection{Construction with common factor}

The solution ${ }^{15}$ consists in constructing $G_{0}$ and $H_{0}$ following these expressions

$$
\begin{aligned}
& H_{0}(z)=(1+1 / z)^{M} Q_{L, M}(z) D_{L}(z), \\
& G_{0}(z)=(1+1 / z)^{M} Q_{L, M}(z) D_{L}(1 / z) z^{-L} .
\end{aligned}
$$

The term in $(1+1 / z)^{M}$ is controling the number of null moments for the filters equal to $M$. The factor $D_{L}$ is the $z$-transform of a FIR causal filter of size $L, D_{L}(z)=1+\sum_{\ell=1}^{L} d_{L}(\ell) z^{-\ell}$. This term controls the property of quasi-analyticity. Then $D_{L}$ is chosen such that the wavelets satisfy approximation (2). A possible solution is given ${ }^{15}$

$$
\frac{\mathrm{e}^{-\mathrm{i} \omega L} D_{L}\left(\mathrm{e}^{-\mathrm{i} \omega}\right)}{D_{L}\left(\mathrm{e}^{\mathrm{i} \omega}\right)}=\mathrm{e}^{-\mathrm{i} \omega / 2}+o_{\omega \rightarrow 0}\left(|\omega|^{2 L+1}\right) .
$$


An explicit expression of the coefficients $d_{L}(\ell), \ell=1, \ldots, L$ is easily obtained. ${ }^{15}$ In the following proposition, an explicit and simple expression of $D_{L}$ is derived.

Proposition 2.1. Let $L$ be a positif integer and let define $D_{L}(z)=1+\sum_{\ell=1}^{L} d_{L}(\ell) z^{-\ell}$ satisfying (5). Then, for all $z \in \mathbb{C}^{*}$,

$$
D_{L}(z)=\frac{z^{-L}}{4 L+2}\left[(1+\sqrt{z})^{2 L+1}+(1-\sqrt{z})^{2 L+1}\right]
$$

where $\sqrt{z}$ is one of the complex root of $z$.

Proof. [Proof of Proposition 2.1] Notice that $d(L)^{-1} z^{L} D_{L}(z)=\sum_{n=0}^{L} \frac{d(L-n)}{d(L)} z^{n}$ and that for all $n=$ $0, \ldots, L-1$,

$$
\begin{aligned}
\frac{d(L-n)}{d(L)} & =\left(\begin{array}{l}
L \\
n
\end{array}\right) \prod_{\ell=L-n}^{L-1} \frac{2 \ell+3}{2 L-2 \ell-1} \\
& =\left(\begin{array}{l}
L \\
n
\end{array}\right)\left(\prod_{k=1}^{n}(2 k-1)\right)^{-1} \prod_{\ell=L-n+1}^{L}(2 \ell+1) \\
& =\frac{L !}{n !(L-n) !} \frac{2^{n} n !}{(2 n) !} \frac{(2 L+1) !}{2^{L} L !} \frac{2^{L-n}(L-n) !}{(2 L-2 n+1) !} \\
& =\left(\begin{array}{c}
2 L+1 \\
2 n
\end{array}\right)
\end{aligned}
$$

It is then easy to check that

$$
d(L)^{-1} z^{L} D_{L}(z)=\frac{1}{2}\left(\left(1+z^{1 / 2}\right)^{2 L+1}+\left(1-z^{1 / 2}\right)^{2 L+1}\right) .
$$

The fact that $d(L)=1 /(2 L+1)$ concludes the proof. $\square$

\subsection{Existence}

A desired property for wavelets may be the property of perfect reconstruction ${ }^{1}$ (and references therein). For filters $H_{0}$ and $G_{0}$ defined by (3) and (4), the perfect reconstruction is satisfied if and only if

$$
\begin{aligned}
R_{L, M}(z) S_{L, M}(z) & +R_{L, M}(-z) S_{L, M}(-z)=2, \\
\quad \text { with } R_{L, M}(z) & =Q_{L, M}(z) Q_{L, M}(1 / z) \\
\text { and } S_{L, M}(z) & =(2+z+1 / z)^{M} D_{L}(z) D_{L}(1 / z) .
\end{aligned}
$$

Our first contribution is to show that there exists a solution of (7), with the common-factor properties (3)-(4). THEOREM 2.2. Let $L$ and $M$ be two positive integer. Then, there exists $Q_{L, M}$ such that $Q_{L, M}(1 / z)$ is a real polynom of $z$ and such that $R_{L, M}(z)=Q_{L, M}(z) Q_{L, M}(1 / z)$ is a solution of (7).

This theorem ensures the existence of a solution for the equations (3)-(4)-(7). Unfortunately, no explicit formula of $R_{L, M}$ and $Q_{L, M}$ is available. However, the proof is constructive and a numerical solution can be derived. An algorithm is proposed, ${ }^{3}$ without guarantee of success.

\subsection{Quality of the approximation}

With the expression obtained in Proposition 2.1, an explicit expression is available to quantify the quality of the analyticity approximation (1) for the pair $\left(\psi_{G}, \psi_{H}\right)$. Let $\left(\phi_{H}(\cdot), \psi_{H}(\cdot)\right)$ be respectively the father and the mother wavelets associated with the (low-pass) wavelet filter $H_{0}$. And let $\left(\phi_{G}(\cdot), \psi_{G}(\cdot)\right)$ denote the father 
and the mother wavelets associated with the wavelet filter $G_{0}$. Recall that their Fourier transforms are given by

$$
\begin{aligned}
& \widehat{\phi}_{H}(\omega)=\prod_{j=1}^{\infty}\left[2^{-1 / 2} H_{0}\left(\mathrm{e}^{\mathrm{i} 2^{-j} \omega}\right)\right], \\
& \widehat{\psi}_{H}(\omega)=2^{-1 / 2} H_{1}\left(\mathrm{e}^{\mathrm{i} \omega / 2}\right) \widehat{\phi}_{H}(\omega / 2),
\end{aligned}
$$

where $H_{1}$ is the corresponding high-pass filter transfer function defined by $H_{1}(z)=z^{-1} H_{0}\left(-z^{-1}\right) \cdot{ }^{15}$ Similar equations hold for $\widehat{\phi}_{G}$ and $\widehat{\psi}_{G}$, using $G_{0}$ and $G_{1} \cdot{ }^{15}$ The expression of the analyticity approximation is given by the following proposition.

Proposition 2.3. For all $\omega \in \mathbb{R}$,

$$
\begin{aligned}
& \widehat{\phi}_{G}(\omega)=\mathrm{e}^{-\mathrm{i} \omega / 2} \mathrm{e}^{\mathrm{i} \beta_{L}(\omega)} \widehat{\phi}_{H}(\omega) \\
& \widehat{\psi}_{G}(\omega)=\mathrm{i} \mathrm{e}^{-\mathrm{i} \eta_{L}(\omega)} \widehat{\psi}_{H}(\omega) .
\end{aligned}
$$

with

$$
\begin{aligned}
& \beta_{L}(\omega)=2(-1)^{L} \sum_{j=1}^{\infty} \operatorname{atan}\left(\tan ^{2 L+1}\left(\omega 2^{-j-2}\right)\right) \\
& \eta_{L}(\omega)=2(-1)^{L} \operatorname{atan}\left(\tan ^{2 L+1}(\omega / 8+\pi / 4)\right)+\beta_{L}(\omega / 2)
\end{aligned}
$$

Consequently,

with $u_{L}(\omega)=1-\mathrm{e}^{-\mathrm{i} \eta_{L}(\omega)}$.

$$
\widehat{\psi}_{H}(\omega)+i \widehat{\psi}_{G}(\omega)=u_{L}(\omega) \widehat{\psi}_{H}(\omega),
$$

Proof. [Proof of Proposition 2.3]

To prove proposition 2.3 , it is sufficient to derive an explicit formula for the ratio $\frac{D_{L}\left(\mathrm{e}^{-\mathrm{i} \omega}\right)}{D_{L}\left(\mathrm{e}^{\mathrm{i} \omega}\right)}$, for all $z=\mathrm{e}^{\mathrm{i} \omega}$ with $\omega \in \mathbb{R}$

$$
\mathrm{e}^{-\mathrm{i} \omega L} \frac{D_{L}\left(\mathrm{e}^{-\mathrm{i} \omega}\right)}{D_{L}\left(\mathrm{e}^{\mathrm{i} \omega}\right)}=\mathrm{e}^{-\mathrm{i} \omega / 2+\mathrm{i} \alpha_{L}(\omega)},
$$

where $\alpha_{L}$ is the function defined on $\mathbb{R}$ by $\alpha_{L}(\omega)=2(-1)^{L} \operatorname{atan}\left(\tan ^{2 L+1}(\omega / 4)\right)$

By denoting $z^{1 / 2}$ any of the two roots of $z$, we observe that, for all $z \in \mathbb{C}^{*}$,

$$
z^{-L} \frac{D_{L}(1 / z)}{D_{L}(z)}=z^{L} \frac{\left(1+z^{-1 / 2}\right)^{2 L+1}+\left(1-z^{-1 / 2}\right)^{2 L+1}}{\left(1+z^{1 / 2}\right)^{2 L+1}+\left(1-z^{1 / 2}\right)^{2 L+1}}=z^{-1 / 2} \frac{\left(1+z^{1 / 2}\right)^{2 L+1}+\left(z^{1 / 2}-1\right)^{2 L+1}}{\left(1+z^{1 / 2}\right)^{2 L+1}-\left(z^{1 / 2}-1\right)^{2 L+1}} .
$$

Set now $z=\mathrm{e}^{\mathrm{i} \omega}$. We deduce that

$$
\mathrm{e}^{-\mathrm{i} \omega L} \frac{D_{L}\left(\mathrm{e}^{-\mathrm{i} \omega}\right)}{D_{L}\left(\mathrm{e}^{\mathrm{i} \omega}\right)}=\mathrm{e}^{-\mathrm{i} \omega / 2} \frac{\mathrm{e}^{\mathrm{i} \omega(2 L+1) / 4} \cos (\omega / 4)^{2 L+1}\left(1+\mathrm{i}(-1)^{L} \tan (\omega / 4)^{2 L+1}\right)}{\mathrm{e}^{\mathrm{i} \omega(2 L+1) / 4} \cos (\omega / 4)^{2 L+1}\left(1-\mathrm{i}(-1)^{L} \tan (\omega / 4)^{2 L+1}\right)} .
$$

The result then follows from the classical result $\frac{1+\mathrm{i} a}{1-\mathrm{i} a}=\mathrm{e}^{2 \mathrm{i} a \tan (a)}$ with here $a=(-1)^{L} \tan (\omega / 4)^{2 L+1}$.

Figure 1 illustrates the behaviour of $u_{L}$. On the intervals $(-4 \pi, 0)$ and $(0,4 \pi)$, it is easy to show that $u_{L}(\omega)$ converges respectively to 0 and 2 when $L$ goes to infinity.

The analyticity of the pair $\left(\widehat{\psi}_{G}, \widehat{\psi}_{H}\right)$ is then obtained thanks to the behaviour of $\widehat{\psi}_{H}(\omega)$. First, $\widehat{\psi}_{H}(\omega) \rightarrow 0$ when $\omega \rightarrow 0$ thanks to the property of the null moments given by (3)-(4). For $|\omega|>4 \pi$, a decreasing property of $\left|\widehat{\psi}_{H}(\omega)\right|$ allows us to verify the analyticity approximation. This last point is verified numerically, as illustrated in Figure 2. 
Figure 1. Analytic approximation. The figure displays $\omega \mapsto\left|u_{L}(\omega)\right|$ for $L=2,5,10$.
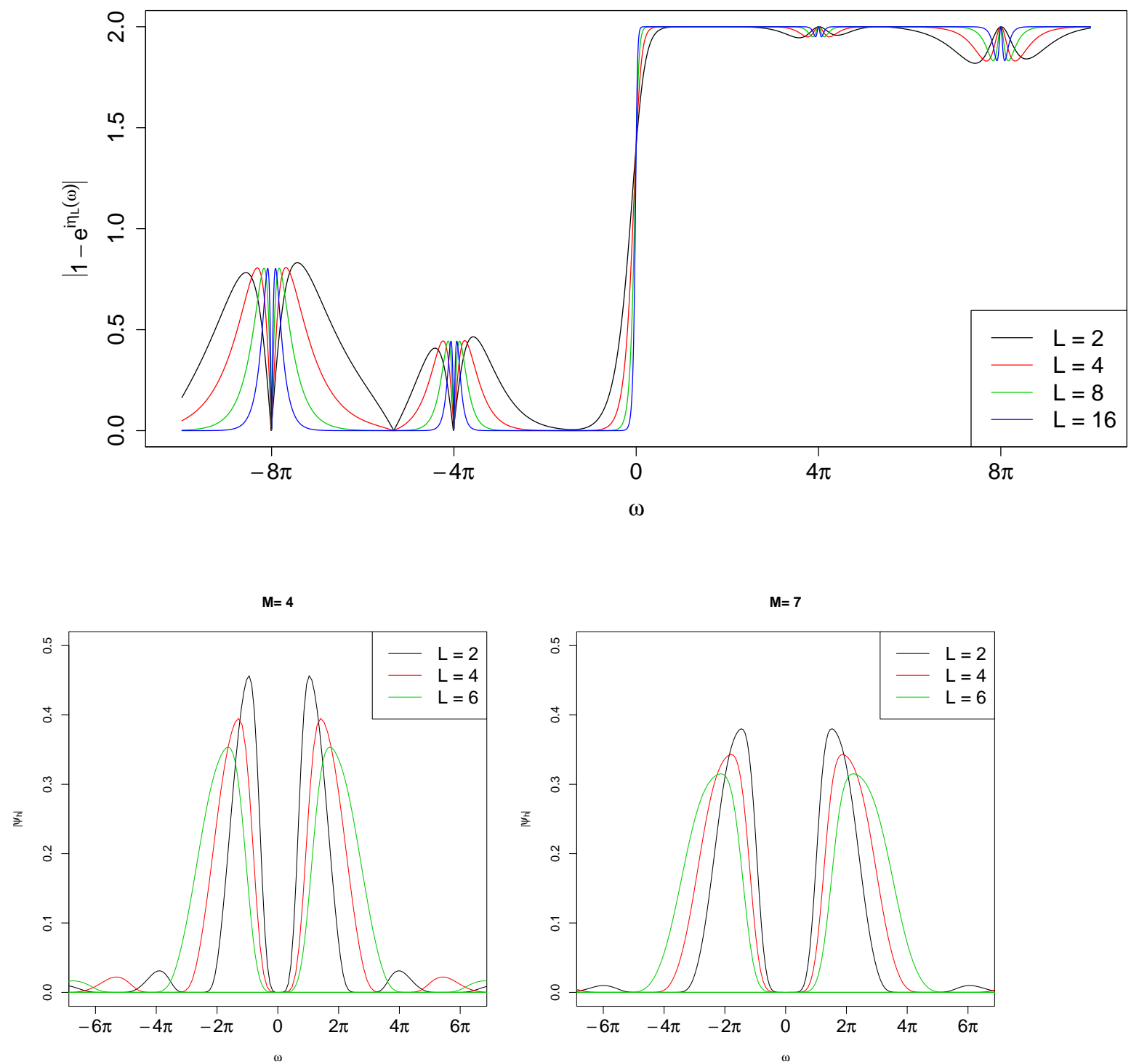

Figure 2. Plot of $\left|\widehat{\psi}_{H}(\lambda)\right|$ for different values of $M$ and $L$, with $Q_{L, M}$ mid-phase factorisations of $R_{L, M}$ in Theorem 2.2.

\section{APPLICATION TO MULTIVARIATE LONG MEMORY PROCESSES $M(\mathbf{d})$}

In order to illustrate the usefulness of the approximately analytic wavelets with common factor, we choose to show their behaviour for $M(\mathbf{d})$ processes with different values of $\mathbf{d}$. In particular, these wavelets extract correlations that are cancelled by real wavelets. From now on, the parameters $M$ and $L$ are chosen such that $M=4$ et $L=2$.

\subsection{Definition of the model}

As an example, we simulate a bivariate $\operatorname{ARFIMA}(0, \mathbf{d}, 0)$ process defined as

$$
X_{\ell}(k)=(1-\mathbb{L})^{-d_{\ell}} u_{\ell}(k), \quad \ell=1,2, k \in \mathbb{Z},
$$


where $\mathbb{L}$ is a lag operator and $\left(\begin{array}{l}u_{1}(k) \\ u_{2}(k)\end{array}\right)$ i.i.d. with law

$$
\mathcal{N}\left(\left(\begin{array}{l}
0 \\
0
\end{array}\right), \Omega\right), \text { avec } \Omega=\left(\begin{array}{cc}
1 & 0.8 \\
0.8 & 1
\end{array}\right) .
$$

Using this model, the spectral density of $\left(X_{1}, X_{2}\right)$ satisfies

$$
\boldsymbol{f}(\lambda)=\boldsymbol{\Lambda}(\mathbf{d}) \mathbf{\Omega} \boldsymbol{\Lambda}(\mathbf{d})^{*} \text { where } \boldsymbol{\Lambda}(\mathbf{d})=\operatorname{diag}\left(\left(1-\mathrm{e}^{-\mathrm{i} \lambda}\right)^{-\mathbf{d}}\right) .
$$

A first order approximation is obtained, when $\lambda \rightarrow 0^{+}$,

$$
\begin{gathered}
f(\lambda) \sim \Sigma(\lambda) \\
\Sigma(\lambda)=\widetilde{\Lambda}(\mathbf{d})^{*} \boldsymbol{\Omega} \widetilde{\Lambda}(\mathbf{d}), \text { with } \widetilde{\Lambda}(\mathbf{d})=\operatorname{diag}\left(\lambda^{-\mathbf{d}} \mathrm{e}^{-\mathrm{i} \pi \mathbf{d} / 2}\right),
\end{gathered}
$$

where the * exponent denotes the complex conjugate. When $\lambda \rightarrow 0^{-}, f(\lambda) \sim \Sigma^{*}(\lambda)$.

Let $\rho$ be the correlation coefficient of $\Sigma(\lambda), \rho=\Sigma_{1,2}(\lambda) / \sqrt{\Sigma_{1,1}(\lambda) \Sigma_{2,2}(\lambda)}=\Omega_{1,2} \mathrm{e}^{\mathrm{i} \pi\left(d_{1}-d_{2}\right) / 2}$ (this does not depend on $\lambda$ ).

In this particular case, the phase of the correlation in $0^{+}$is equal to $\pi\left(d_{1}-d_{2}\right) / 2$. The discontinuity of the spectral density in 0 perfectly justifies the use of analytic transforms for the identification of the phase. Indeed, it is crucial to consider only the positive (or negative) frequency in order to keep the information on the phase.

Define $\left\{W_{j, k}(\ell), j \geqslant 0, k \in \mathbb{Z}\right\}$ for $\ell=1,2$ the wavelet coefficients of the process $X_{\ell}$ obtained from the wavelet transform $\psi$ :

$$
W_{j, k}(\ell)=\int_{\mathbb{R}} \widetilde{X}_{\ell}(t) \psi_{j, k}(t) d t \quad j \geqslant 0, k \in \mathbb{Z},
$$

where $\psi_{j, k}(\cdot)=2^{-j / 2} \psi\left(2^{-j} \cdot-k\right)$ et $\widetilde{X}_{\ell}(t)=\sum_{k \in \mathbb{Z}} X_{\ell}(k) \phi(t-k)$. Let $\theta_{j}$ denotes the empirical correlation between $\left\{W_{j, k}(1), k \in \mathbb{Z}\right\}$ and $\left\{W_{j, k}(2), k \in \mathbb{Z}\right\}$, that is,

$$
\theta_{j}=\operatorname{Cor}\left(\left\{\mathbf{W}_{j, k}(1), k \in \mathbb{Z}\right\},\left\{\mathbf{W}_{j, k}(2), k \in \mathbb{Z}\right\}\right)
$$

for a given scale $j \geqslant 0$. The behaviour of $\theta_{j}$ when $j \rightarrow \infty$ is intrisically linked to $\rho$. More precisely, when $\psi$ is real, Proposition $2^{14}$ shows that asymptotically $\theta_{j}$ tends to the real part of $\rho$ when $j$ tends to infinity. In particular, when the phase is equal to $\pi / 2$, i.e. when $d_{1}-d_{2}$ is close to 1 modulo 2 , the correlation of the real wavelet coefficients is equal to 0 . When $\psi$ is analytic, $\left\{\theta_{j}, j \geqslant 0\right\}$ are no more real and their imaginary part can yield additional information about the phase. It is shown in this paper on simulations that $\rho$ can be recovered thanks to $\left\{\theta_{j}, j \geqslant 0\right\}$ when taking into account the imaginery part. An interesting perspective is to build an estimation procedure of $\rho$ based on this observation. In the following, Re indicates the real part and $I m$ is the imaginary part.

\subsection{Numerical results}

For the first simulation, the long memory parameter $\mathbf{d}$ is equal to $(0.2,0.2)$. The phase of the $M(\mathbf{d})$ process defined above, is equal to 0 . Figure 3 displays $\left\{\theta_{j}, j \geqslant 0\right\}$ with real wavelets computed using time series of size $2^{12}$. 1000 simulations were repeated to obtain the boxplots. This figure is clearly showing that $\rho$ is recovered directly from the correlation of the wavelet coefficients. For the second simulation, the long memory parameter $\mathbf{d}$ is equal to $(0.2,1.2)$. The phase is here equal to $\pi / 2$ and the approximation of the spectral density at the zero frequancy contains only the imaginary part. In this example, Figure 3 illustrates the cancellation of the correlation of the wavelet coefficients and consequently the impossibility to identify $\rho$. Indeed, only the imaginary part of $f$ contains information in this example.

In the first simulation, with $d_{1}=d_{2}=0.2$, when using an analytic wavelets, Figure 4 shows similar results as for real wavelets. In addition, the imaginary part of the correlations $\left\{\theta_{j}, j \geqslant 0\right\}$ is equal to 0 . In 
Figure 3. Boxplot of the empirical correlations of the wavelet coefficients $\left\{\theta_{j}, j \geqslant 0\right\}$ for real wavelets in terms of the scales. In red, the real part of the theoretical value of $\rho$ of the ARFIMA model.
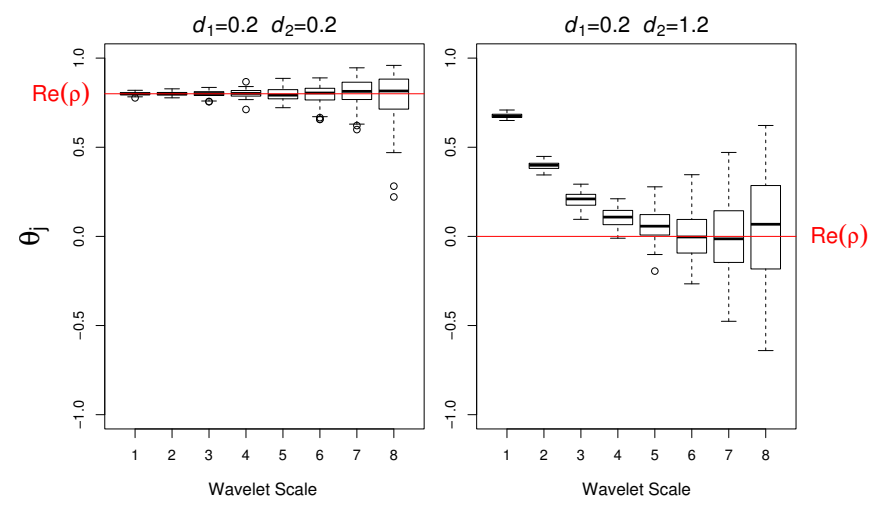

Figure 4. Boxplot of the empirical correlations of the wavelet coefficients $\left\{\theta_{j}, j \geqslant 0\right\}$ for analytic wavelets in terms of the scales. In red, the real part of the thoretical value of $\rho$ of the ARFIMA model.
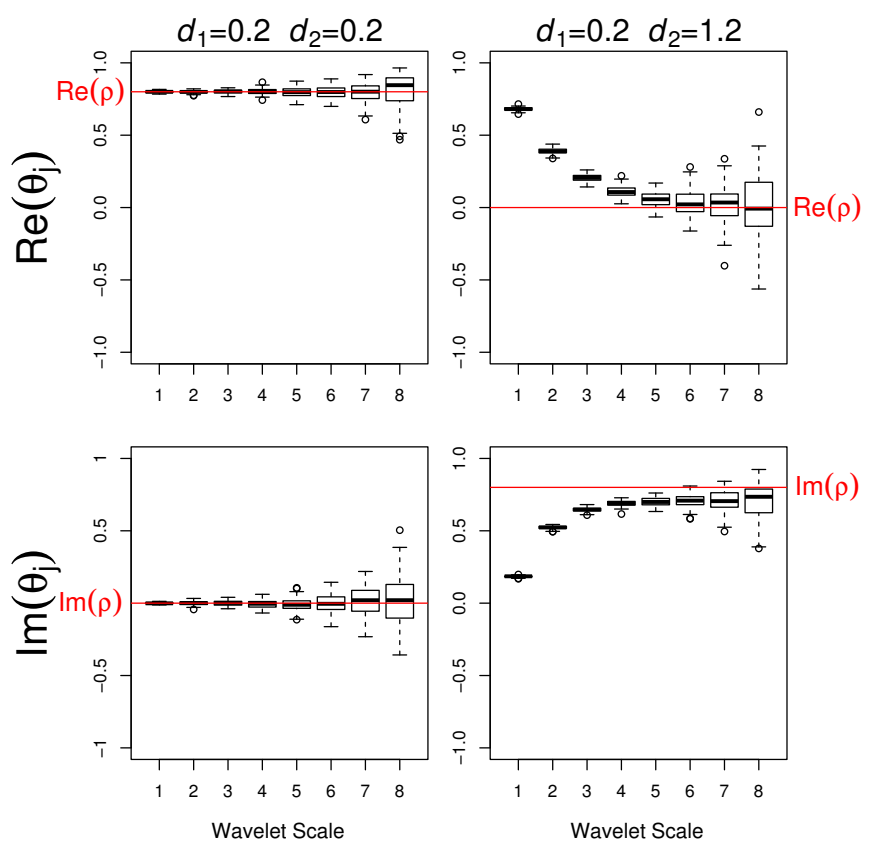

the second simulation, when $d_{1}=0.2$ et $d_{2}=1.2$, Figure 4 reveals that the real part of the correlations $\left\{\theta_{j}, j \geqslant 0\right\}$ is vanishing after a certain scale as it is observed with real wavelets. However, the imaginary part of the wavelet coefficient correlations is, as expected in this case, converging in mean to $\rho$.

Other simulation studies bring to light a similar conlusion, that is, the empirical correlations of wavelet coefficents based on approximately analytic wavelets enable to recover the quantity $\rho$. Considering both real and imaginary parts of the quasi-analytic wavelet coefficients, it enables us to identify the modulus and phase of the spectral density at the $0^{+}$frequency. They are not presented in the paper for concision. 


\section{conclusion}

In this paper, our first contribution is to show the existence of the approximately analytic wavelets using a common-factor design. ${ }^{3}$ An example of the usefulness of analytic wavelets is described in the context of analysing multivariate processes. The results presented in this paper suggest directions for future investigations. Theoretical results on the quality of the analytic approximation are needed (see the paper in preparation?). Indeed, this is necessary to derive statistical properties of the estimation of the parameters of the multivariate long-memory model. ${ }^{14}$ Also a numerical instability is observed when varying the parameters $L$ and $M$, this would be studied in a future work.

\section{REFERENCES}

[1] Selesnick, I. W., Baraniuk, R. G., and Kingsbury, N. C., "The dual-tree complex wavelet transform," Signal Processing Magazine, IEEE 22(6), 123-151 (2005).

[2] Thiran, J.-P., "Recursive digital filters with maximally flat group delay," Circuit Theory, IEEE Transactions on 18(6), 659-664 (1971).

[3] Selesnick, I. W., "The design of approximate hilbert transform pairs of wavelet bases," Signal Processing, IEEE Transactions on 50(5), 1144-1152 (2002).

[4] Gençay, R., Selçuk, F., and Whitcher, B. J., [An introduction to wavelets and other filtering methods in finance and economics ], Academic Press (2001).

[5] Nielsen, M. Ø. and Frederiksen, P. H., "Finite sample comparison of parametric, semiparametric, and wavelet estimators of fractional integration," Econometric Reviews 24(4), 405-443 (2005).

[6] Abry, P. and Veitch, D., "Wavelet analysis of long-range-dependent traffic," Information Theory, IEEE Transactions on 44(1), 2-15 (1998).

[7] Percival, D. B. and Walden, A. T., [Wavelet methods for time series analysis ], vol. 4, Cambridge University Press (2006).

[8] Papanicolaou, G. C. and Sølna, K., "Wavelet based estimation of local Kolmogorov turbulence," Theory and Applications of Long-range Dependence, 473-505 (2003).

[9] Whitcher, B. and Jensen, M. J., "Wavelet estimation of a local long memory parameter," Exploration Geophysics 31(1/2), 94-103 (2000).

[10] Maxim, V., Şendur, L., Fadili, M. J., Suckling, J., Gould, R., Howard, R., and Bullmore, E. T., "Fractional Gaussian noise, functional MRI and Alzheimer's disease," NeuroImage 25, 141-158 (2005).

[11] Faÿ, G., Moulines, E., Roueff, F., and Taqqu, M. S., "Estimators of long-memory: Fourier versus wavelets," Journal of Econometrics 151(2), 159-177 (2009).

[12] Shimotsu, K., "Gaussian semiparametric estimation of multivariate fractionally integrated processes," Journal of Econometrics 137(2), 277-310 (2007).

[13] Kechagias, S. and Pipiras, V., "Definitions and representations of multivariate long-range dependent time series," Journal of Time Series Analysis (2014).

[14] Achard, S. and Gannaz, I., "Multivariate wavelet whittle estimation in long-range dependence," Journal of Time Series Analysis 37(4), 476-512 (2016).

[15] Selesnick, I. W., “Hilbert transform pairs of wavelet bases," Signal Processing Letters, IEEE 8(6), 170-173 (2001). 